\title{
Anti-oxidative effects of exogenous ganglioside GD1a and GT1b on embryonic developmental competence in pigs
}

\author{
Jin-Woo Kim ${ }^{1,2, \#}$, Hyo-Jin Park ${ }^{1,2, \#}$, Seul-Gi Yang ${ }^{1,2}$ and Deog-Bon Koo ${ }^{1,2, *}$ \\ ${ }^{1}$ Department of Biotechnology, College of Engineering, Daegu University, Gyeongsan 38453, Korea \\ ${ }^{2}$ Institute of Infertility, Daegu University, Gyeongsan 38453, Korea
}

Received December 4, 2020

Revised December 18, 2020

Accepted December 18, 2020

\section{*Correspondence \\ Deog-Bon Koo \\ E-mail: dbkoo@daegu.ac.kr}

ORCID

https://orcid.org/0000-0001-7825-9598

"The first two authors contributed equally to this work.

\begin{abstract}
Gangliosides are glycolipids in which oligosaccharide is combined with sialic acids. Our previous studies have suggested an interplay between ganglioside GD1a/GT1b and meiotic maturation capacity in porcine oocyte maturation. Furthermore, ganglioside GD1a and GT1b are known for its antioxidant activity, but it is still unclear whether possible antioxidant role of GD1a and GT1b is involved in porcine embryos development competence during in vitro culture (IVC). Here, the effects of ganglioside GD1a and GT1b on the embryonic developmental competence during in vitro culture of porcine were investigated. The effects of ganglioside GD1a and GT1b on the expression of ST3GAL2 were confirmed during embryos development (2-cell, 4-cell, 8-cell and blastocyst) using immunofluorescent staining (IF). As a result, the fluorescent expression of ST3GAI2 was higher in embryos at 4-8 cells stage than blastocysts. Blastocyst development rate significantly increased in only 0.1 $\mu \mathrm{M}$ GD1a and GT1b treated groups compared with control group. To investigate the cellular apoptosis, we analyzed TUNEL assay. In case of only $0.1 \mu \mathrm{M}$ GD1a and GT1b treated groups, the total number of cells in blastocyst compared with control group, but there was no significant difference in the rate of apoptotic cells. We identified the intracellular ROS levels using DCF-DA staining. According to the result, ROS production significantly decreased in blastocysts derived from the $0.1 \mu \mathrm{M}$ GD1a and GT1b treated groups. These results suggest that ganglioside GD1a and GT1b improve the developmental competence of porcine embryos via reduction of intracellular ROS during preimplantation stage.
\end{abstract}

Keywords: GD1a, GT1b, In vitro culture (IVC), porcine embryo, reactive oxygen species (ROS)

\section{INTRODUCTION}

The in vitro production (IVP) of porcine embryos is an important technology for agricultural and biomedical animal models, including development of advanced reproductive technologies (ARTs), embryos transfer, vit- rification, and production of transgenic animals (Prather et al., 2003). However, the in vitro culture systems still have many problems, such as embryos development arrest and fragmentation (Chen et al., 2018). It is difficult to mimic the complex environment of mammalian uteri and oviducts in vitro. Furthermore, in vitro derived porcine 
blastocysts have low development rate and quality of blastocysts compared with in vivo derived porcine blastocysts (Rath et al., 1995; Macháty et al., 1998). In order to solve these problem, natural materials, nutrients, and various antioxidants were added to improve the quality of blastocysts (Lee et al., 2015; Yang et al., 2018).

During respiration and cellular metabolism in mammalian cells, reactive oxygen species (ROS) such as superoxide anion radical $\left(\mathrm{O}_{2} \cdot{ }^{-}\right)$, hydrogen peroxide $\left(\mathrm{H}_{2} \mathrm{O}_{2}\right)$, and hydroxyl radical $(\cdot \mathrm{OH})$ are produced. Maintaining homeostasis of ROS is critical for cell growth, proliferation, division, and survival (Covarrubias et al., 2008). Excessive ROS induced oxidative stress (OS) occurs due to an imbalance between the production of ROS and the antioxidant reaction that remove ROS. Many studies have demonstrated that oxidative damage to preimplantation embryos is harmful in various species, such as human (Yang et al., 1998), cow (Favetta et al., 2007), and mouse (Goto et al., 1993). Furthermore, our previous studies have reported increase in efficiency of IVP embryos using antioxidant and natural substances to suppress ROS (Lee et al., 2015; Yang et al., 2018). However, no reports have investigated this possible interaction between GD1a/GT1b and antioxidative effects response to ROS in porcine embryos.

Glycosphingolipids are divided into neutral glycosphingolipids and acidic glycosphingolipids. Acidic glycosphingolipids containing sialic acid residues in carbohydrate moiety are referred to as gangliosides. Gangliosides are found in the central nervous system and vertebrate plasma membranes (Kwak et al., 2011). Gangliosides are known to play a critical role in cellular functions, such as cell growth, differentiation, and signaling (Kim et al., 2008). Ganglioside GD1a and GT1b are synthesized in GM1a and GD1ab by the ST3GAL2 enzyme (Sturgill et al., 2012). GD1a and GT1b are known for being involved in cell survival and signaling pathways in cancer and neuron. In mice, GT1b was reported to be expressed during embryonic development (Kim et al., 2008). Also, after freezing and thawing, GT1b was expressed in surviving embryos, but GT1b was not expressed in dead embryos (Kim et al., 2008). In addition, GT1b was reported to protect sperm against $\mathrm{H}_{2} \mathrm{O}_{2}$-mediated cellular damage in human spermatozoa (Gavella et al., 2012; Gavella et al., 2013), and to reduce ROS during in vitro maturation (IVM) of porcine oocytes (Hwang et al., 2015). GD1a has been reported to increase the survival rate of PC12 cell and reduce ROS in- duced by LPS (Nikolaeva et al., 2015). However, there are no known effects of GD1a and GT1b on embryos development competence in porcine zygote. The aim of the present study was to determine whether the addition of GD1a and GT1b to culture medium affects embryonic developmental competence and quality of blastocysts through the reducing ROS production in porcine embryos.

\section{MATERIALS AND METHODS}

\section{Chemicals}

Unless otherwise stated, all chemicals reagents used in this study were purchased from Sigma-Aldrich Korea (Sigma-Aldrich Korea, Yongin, Korea).

\section{In vitro maturation (IVM)}

Porcine ovaries obtained from a local slaughterhouse were transported to the laboratory in $0.9 \%$ saline supplement with $75 \mu \mathrm{g} / \mathrm{mL}$ potassium penicillin $\mathrm{G}$ at $30-35^{\circ} \mathrm{C}$. Cumulus-oocyte complexes (COCs) were aspirated from 3-6 $\mathrm{mm}$ follicle using $10 \mathrm{~mL}$ syringe and 18 gauge needle. Obtained COCs were selected from thick surrounding cumulus cells and equality cytoplasm using mouth pipetting, then were washed three times in Tyrode's lactat$\mathrm{N}-2$ - hydroxyethylpiperazine-N'-2-ethanesulfonic acid (TL-HEPES). Next, 50 immature COCs were matured in $500 \mu \mathrm{L}$ of in vitro maturation medium in four-well dish (Nunc, Roskilde, Denmark) at $38.5^{\circ} \mathrm{C}$ under $5 \% \mathrm{CO}_{2}$ and saturated humidity. BSA-free North Carolina State University (NCSU)-23 medium supplemented with $0.57 \mathrm{mM}$ cysteine, $10 \mathrm{IU} / \mathrm{mL}$ pregnant mare's serum gonadotropin (PMSG), $10 \mathrm{IU} / \mathrm{mL}$ human chorionic gonadotropin (hCG), $25 \mu \mathrm{M} \beta$-mercaptoethanol ( $\beta$-me), $10 \mathrm{ng} / \mathrm{mL}$ EGF, $10 \%$ follicular fluid (v/v) were used for COCs maturation. After $22 \mathrm{~h}$ maturation, COCs were washed three times in PMSG and hCG-free maturation medium and then cultured in medium for $22 \mathrm{~h}$.

\section{In vitro fertilization and culture (IVF \& IVC)}

In current study, embryo development experiment was performed by evaluating the in vitro culture system, according to the methods described by Kim (2016). At the end of maturation period, matured COCs were performed in vitro fertilization. The IVF medium, modified Trisbuffered medium (mTBM), consisted of $113.1 \mathrm{mM} \mathrm{NaCl}$, $3 \mathrm{mM} \mathrm{KCl}, 7.5 \mathrm{mM} \mathrm{CaCl}_{2}, 5 \mathrm{mM}$ sodium pyruvate, $11 \mathrm{mM}$ 
glucose, $20 \mathrm{mM}$ Tris, $2.5 \mathrm{mM}$ caffeine sodium benzoate, and $1 \mathrm{mg} / \mathrm{mL}$ BSA. Cumulus cells in matured COCs were removed using $0.1 \%$ hyaluronidase. Denuded oocytes were washed three times by MTBM and 15-20 oocytes were transferred into $48 \mu \mathrm{L}$ drop of mTBM under mineral oil. Porcine semen was supplied twice a week by AI (Darby pig AI Center, Anseong, Korea) and kept at $17^{\circ} \mathrm{C}$ for $2-3$ days. Semen was washed three times by centrifugation in PBS supplemented with $1 \mathrm{mg} / \mathrm{mL}$ BSA (w/v), $100 \mathrm{mg} / \mathrm{mL}$ penicillin $\mathrm{G}$, and $75 \mathrm{mg} / \mathrm{mL}$ streptomycin sulfate. Then, spermatozoa were made to swim up in mTBM. Then $2 \mu \mathrm{L}$ of spermatozoa were added to $48 \mu \mathrm{L}$ drop give to a final concentration of $1.5 \times 10^{5}$ sperms $/ \mathrm{mL}$. Oocytes were coincubated with spermatozoa for $6 \mathrm{~h}$ at $38.5^{\circ} \mathrm{C}$ under $5 \%$ $\mathrm{CO}_{2}$. Approximately $6 \mathrm{~h}$ after in semination, embryos were cultured in $50 \mu \mathrm{L}$ drops of PZM-3 medium with $3 \mathrm{mg} / \mathrm{mL}$ $\mathrm{BSA}$ at $38.5^{\circ} \mathrm{C}$ under $5 \% \mathrm{CO}_{2}$. After $48 \mathrm{~h}$ of culture, $25-$ 30 cleaved embryos were further cultured in $50 \mu \mathrm{L}$ drops of PZM- 3 medium with $3 \mathrm{mg} / \mathrm{mL}$ BSA at $38.5^{\circ} \mathrm{C}$ under $5 \%$ $\mathrm{CO}_{2}$ for 4 days. Blastocyst formation was evaluated after 6 days of culture. Ganglioside GD1a and GT1b were added in different concentration in culture medium.

\section{Assessment of apoptosis in blastocysts}

Cellular apoptosis analysis was performed by TUNEL assay, according to the methods described by Kim (2016). Apoptotic blastocysts were detected using an In Situ Cell Death Detection Kit (Roche Diagnostics GmbH, Mannheim, Germany) according to the manufacturer's instructions. On day 6 , the blastocysts from IVF were washed with $0.1 \%$ PVA in PBS and then fixed in 4\% PFA in PBS for $1 \mathrm{~h}$ at room temperature. Next, blastocysts were permeabilized using $0.1 \%(\mathrm{v} / \mathrm{v})$ Triton $\mathrm{X}-100$ for $30 \mathrm{~min}$ at $44^{\circ} \mathrm{C}$. The fixed embryos were incubated in TUNEL reaction medium for 1 $\mathrm{h}$ at $38.5^{\circ} \mathrm{C}$, then washed and mounted on slides. Wholemount embryos were examined under an epifluorescence microscope (Olympus, Tokyo, Japan) following TUNEL assay and DAPI staining, and the number of apoptotic nuclei and total number of nuclei were counted.

Table 1. Antibodies used for immunofluorescence

\begin{tabular}{cccc}
\hline Target protein & Dilution & Catalog number & Source* \\
\hline ST3GAL2 & 1:3000 & sc-292044 & Santa Cruz Biotechnology \\
\hline *Santa Cruz Biotechnology, Dallas, TX, USA. &
\end{tabular}

\section{Measurement of ROS levels}

ROS levels was performed by DCF-DA staining, according to the experimental methods described by Kim (2016). The levels of $\mathrm{H}_{2} \mathrm{O}_{2}$ in each embryo were measured using the difluorodihydrofluorescein diacetate method (H2DCFDA; Molecular Probes, Eugene, OR, USA) as described previously (Choi et al., 2008). H2DCFDA produces an intermediate difluorodihydrofluorescein (DCF) after reaction with ROS. Oxidation DCF produces difluorofluorescein, which can be monitored in the fluorescence microscope (Crow et al., 1997). At days 6 after IVF, IVP blastocysts were recovered and used for the experiment. After three times of washing in IVC medium, blastocysts were transferred into IVC medium containing $5 \mathrm{mM} \mathrm{H2D-}$ CFDA for $20 \mathrm{~min}$ at $38.5^{\circ} \mathrm{C}$. A stock solution of H2DCFDA dissolved in dimethylsulfoxide (DMSO) was then diluted in IVC medium, after which the permeabilized blastocysts in H2DCFDA were washed three times with $0.1 \%$ polyvinylalcohol (PVA)-PBS and placed into a $50 \mu \mathrm{L}$ drop covered with mineral oil. The fluorescent emissions from the embryos were recorded with a fluorescent microscope (IX 51; Olympus, Tokyo, Japan) equipped with a cooled charge coupled device (CCD) camera which filters at $488 \mathrm{~nm}$ and
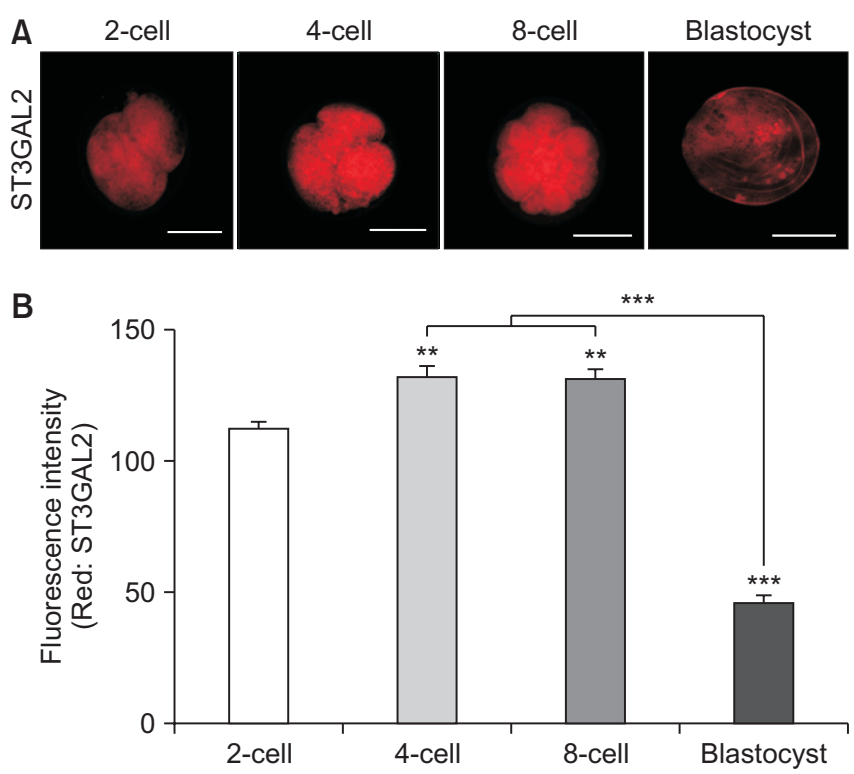

Fig. 1. Fluorescence expression patterns of ST3GAL2 in porcine embryos during IVC. (A) ST3GAL2 expression was examined in embryonic development during IVC by immunofluorescence. (B) Quantitative analysis of ST3GAL2 protein levels in embryonic development during IVC. Data in the bar graph represent the means \pm SEM of three independent experiments. Scale bar $=100 \mu \mathrm{m}$. Differences were considered significant at ${ }^{* *} p<0.01$ and ${ }^{* * *}<0.001$. 
$520 \mathrm{~nm}$ were used for excitation and emission, respectively. The recorded fluorescent images were analyzed by subtracting background and measuring integrated density with Image J software Version 1.38 (National Institutes of Health, Bethesda, MD, USA). Overall, blastocysts in each treatment groups were examined.

\section{Statistical analysis}

All percentage data obtained in this study are presented as the mean \pm standard deviation (SD). Moreover, Western blot experiments were performed in triplicate and all values were presented as the mean \pm standard error of the mean (SEM). The results were analyzed using either a
A
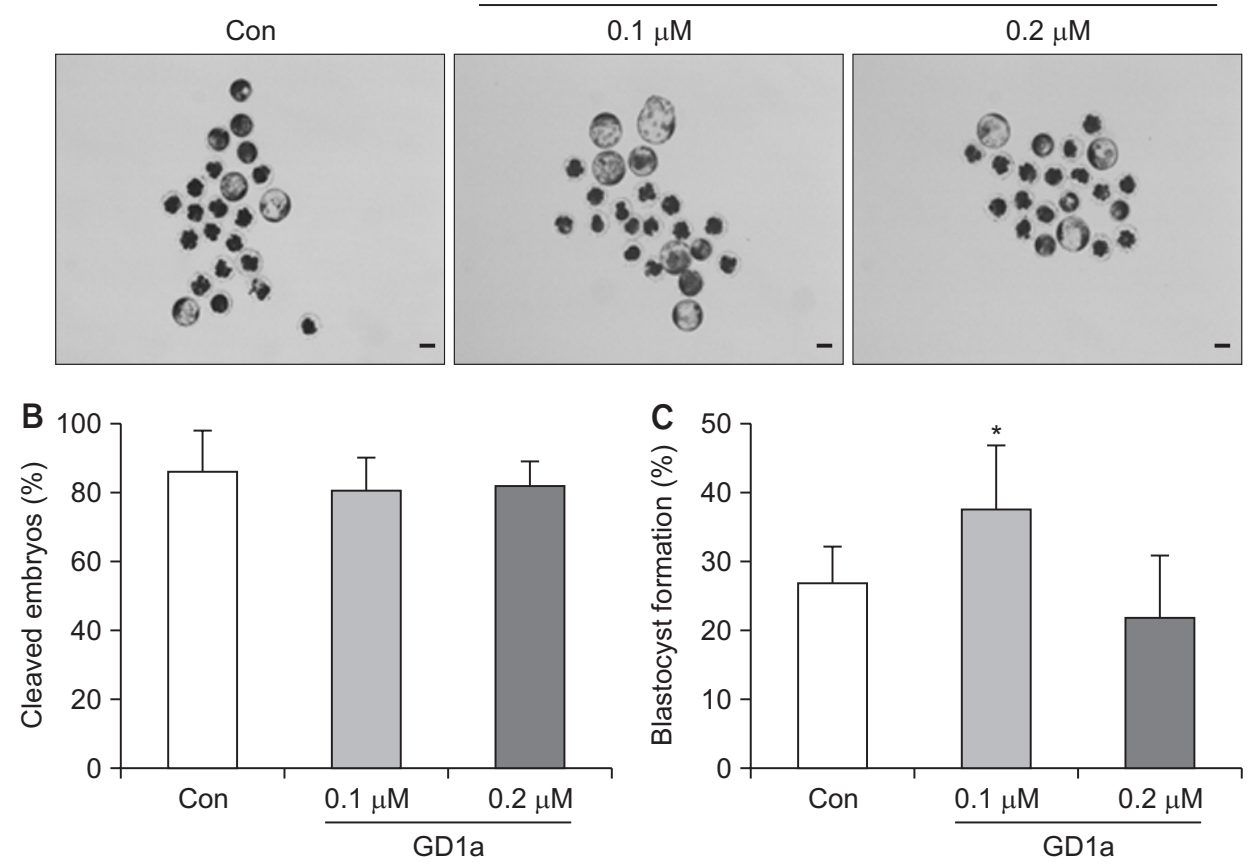

A
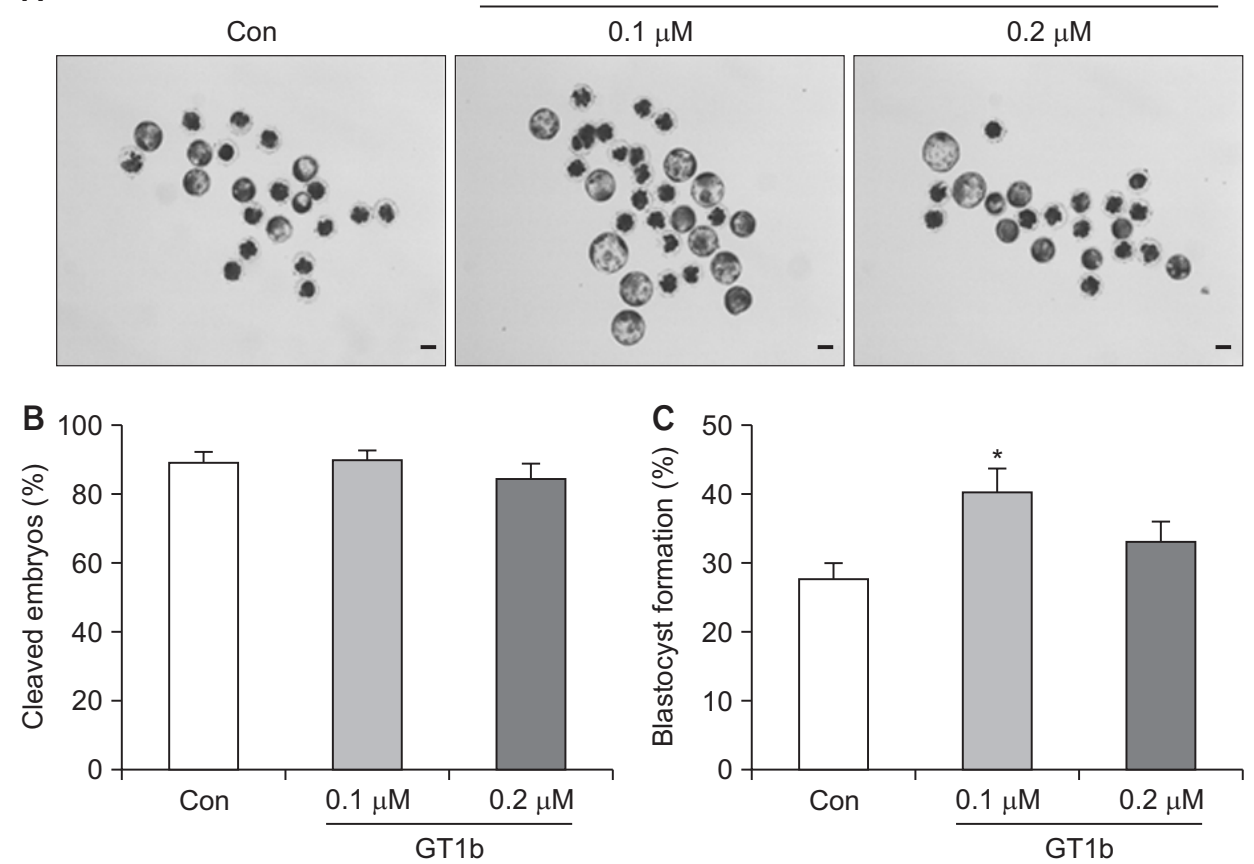

Fig. 2. Effects of ganglioside GD1a during IVC on development of porcine embryos. (A) Representative photographs of blastocysts according to treatment concentrations $(0.1$ and $0.2 \mu \mathrm{M})$ of GD1a. Scale bar $=100 \mu \mathrm{m}$. Rates of cleavage stage (B) and blastocyst stage (C) in the absence or presence of different concentrations of GD1a. Data in the bar graph represent the means \pm SEM of three independent experiments. Differences were considered significant at ${ }^{*} p<0.05$.
Fig. 3. Effects of exogenous ganglioside GT1b during IVC on development of porcine embryos. (A) Representative photographs of blastocysts according to treated concentrations $(0.1$ and 0.2 $\mu \mathrm{M})$ of GT1b. Scale bar $=100 \mu \mathrm{m}$. Rates of cleavage (B) and blastocyst stage (C) in the absence or presence of different concentrations of GT1b. Data in the bar graph represent the means \pm SEM of three independent experiments. Differences were considered significant at ${ }^{*} p<$ 0.05 . 
one-way ANOVA followed by Bonferroni's Multiple Comparison Test or by performing a $t$-test. All data were analyzed using the GraphPad Prism 5.0 software package (San Diego, CA, USA). Differences were considered significant at ${ }^{*} p<0.05, * *<0.01$, and ${ }^{* * * *}<0.001$.

\section{RESULTS}

Expression of ST3GAL2 protein in embryos during IVC We measured the expression of ST3GAL2 (Table 1) as a synthesizing enzyme for GD1a and GT1b production in porcine embryos during IVC process (2-cells, 4-cells, 8 -cells, and blastocysts) by immunofluorescence (IF). As shown in Fig. 1, the expression of ST3GAL2 was significantly increased in 4-8 cells stage than in 2 cells and blastocysts stage. This result demonstrated that the GD1a and GT1b synthesizing enzyme ST3GAL2 was expressed in embryos during development stage.

\section{Effects of ganglioside GD1a and GT1b on embryonic developmental competence in pigs}

To find the appropriate treatment concentration of GD1a and GT1b, we supplied exogenous ganglioside GD1a and GT1b with culture medium for porcine embryos development. After fertilization, porcine zygotes were cultured in IVC medium supplemented with 0.1 , and $0.2 \mu \mathrm{M}$ GD1a or GT1b for 6 days, respectively. As shown in Fig. 2 and 3, the rates of blastocysts formation and expanded blastocysts production in the presence of only 0.1 $\mu \mathrm{M}$ GD1a and GT1b were significantly higher than those of control (Table 2; Non-treatment: $27.0 \pm 5.0 \%$ vs GD1a $0.1 \mu \mathrm{M}: 37.5 \pm 9.5 \%$, Table 3; Non-treatment: $27.5 \pm 6.0 \%$ vs GT1b $0.1 \mu \mathrm{M}: 40.1 \pm 9.0 \%$ ). These results showed the positive roles of GD1a and GT1b on development confidence in porcine embryos.

\section{Cellular apoptosis in blastocysts derived from ganglioside GD1a and GT1b treated embryos}

To investigate the effects of exogenous GD1a and GT1b treatment on quality of blastocysts during IVC, we confirmed apoptotic pattern of porcine blastocysts using TUNEL assay (Fig. 4). As shown Table 4, the number of total nuclei increased in blastocysts derived from GD1a and GT1b treated embryos than in the control group. However, no difference was observed in the number of TUNEL positive nuclei between GD1a or GT1b treated groups and control groups.

\section{Effects of ganglioside GD1a and GT1b on intracellular ROS levels of porcine embryos}

We investigated the intracellular levels of ROS in ganglioside GD1a and GT1b treated embryo at 4-cells, 8-cells, and blastocyst stages. As shown in Fig. 5, the intracellular level of ROS was significantly decreased in blastocysts derived from $0.1 \mu \mathrm{M}$ GD1a and GT1b treated embryos compared with the control group. However, there was no significant difference at 4-cells and 8-cells.

\section{DISCUSSION}

In present study, we demonstrated that exogenous ganglioside GD1a and GT1b enhanced embryonic development competence and blastocysts quality in porcine embryo. In addition, intracellular ROS levels reduced in

Table 2. Effect of various concentrations of GD1a on preimplantation development of porcine embryos in vitro

\begin{tabular}{cccc}
\hline Treatment & No. of embryos examined & \% of embryos cleaved $(\mathrm{n})$ & \% of blastocysts $(\mathrm{n})$ \\
\hline Non-treated & 217 & $86.4 \pm 12.0(182)$ & $27.0 \pm 5.0(56)^{\mathrm{a}}$ \\
GD1a 0.1 $\mu \mathrm{M}$ & 217 & $80.8 \pm 9.5(172)$ & $37.5 \pm 9.5(78)^{\mathrm{b}}$ \\
GD1a 0.2 $\mu \mathrm{M}$ & 217 & $82.3 \pm 6.8(175)$ & $21.9 \pm 9.0(46)^{\mathrm{a}}$ \\
\hline
\end{tabular}

Data are expressed as means \pm SD of three independent experiments. Different superscript letters denote significant differences $(p<0.05)$.

Table 3. Effect of various concentrations of GT1b on preimplantation development of porcine embryos in vitro

\begin{tabular}{cccc}
\hline Treatment & No. of embryos examined & \% of embryos cleaved $(\mathrm{n})$ & \% of blastocysts $(\mathrm{n})$ \\
\hline Non-treated & 197 & $89.1 \pm 7.8(175)$ & $27.5 \pm 6.0(54)^{\mathrm{a}}$ \\
GT1b 0.1 $\mu \mathrm{M}$ & 197 & $89.8 \pm 8.2(178)$ & $40.1 \pm 9.0(79)^{\mathrm{b}}$ \\
GT1b 0.2 $\mu \mathrm{M}$ & 197 & $84.2 \pm 11.9(164)$ & $32.8 \pm 8.1(63)^{\mathrm{a}}$ \\
\hline
\end{tabular}

Data are expressed as means \pm SD of three independent experiments. Different superscript letters denote significant differences $(p<0.05)$. 
A
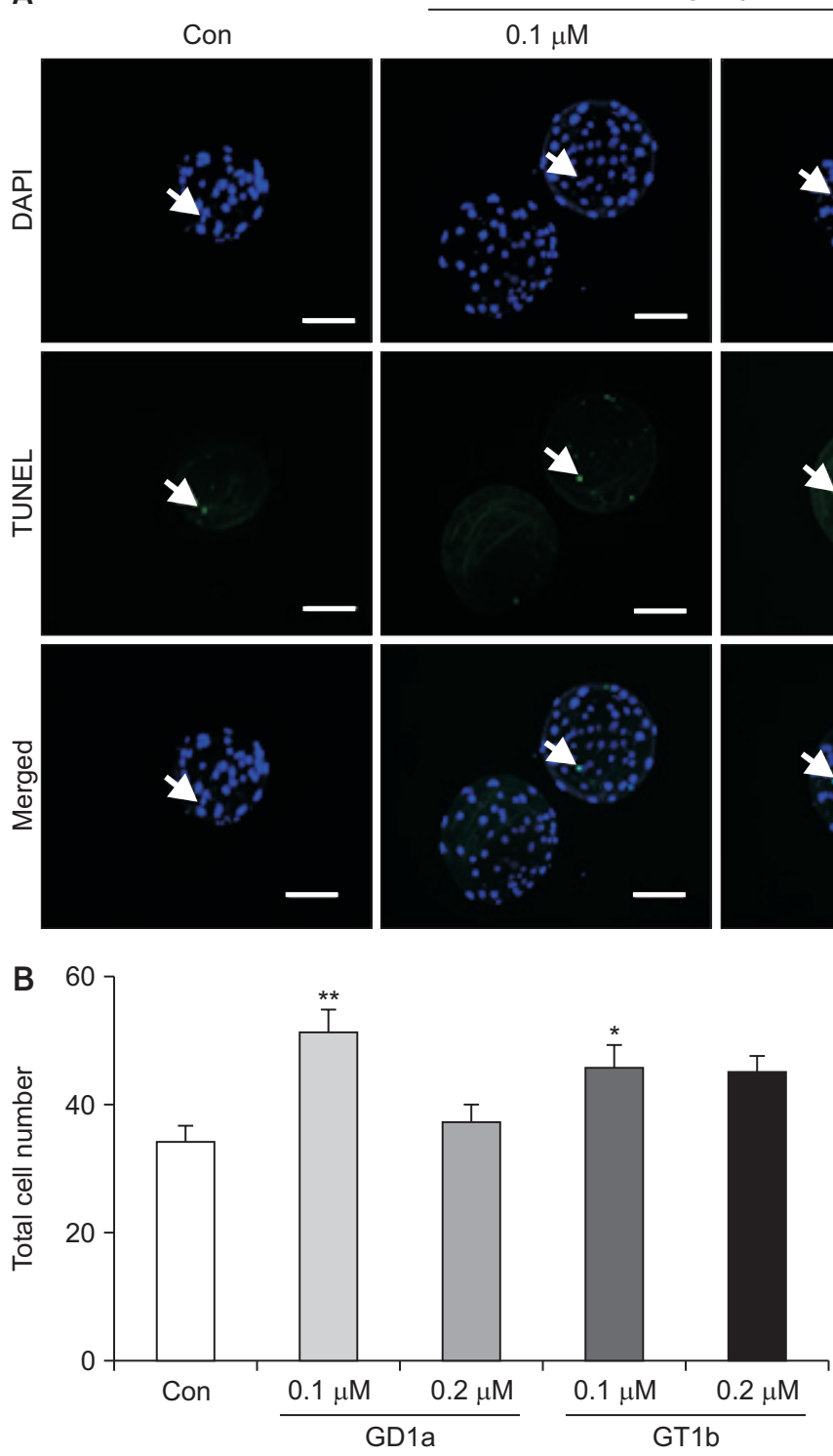

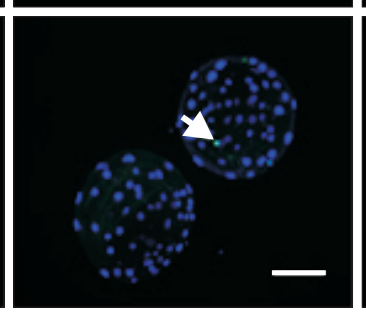

GD1a
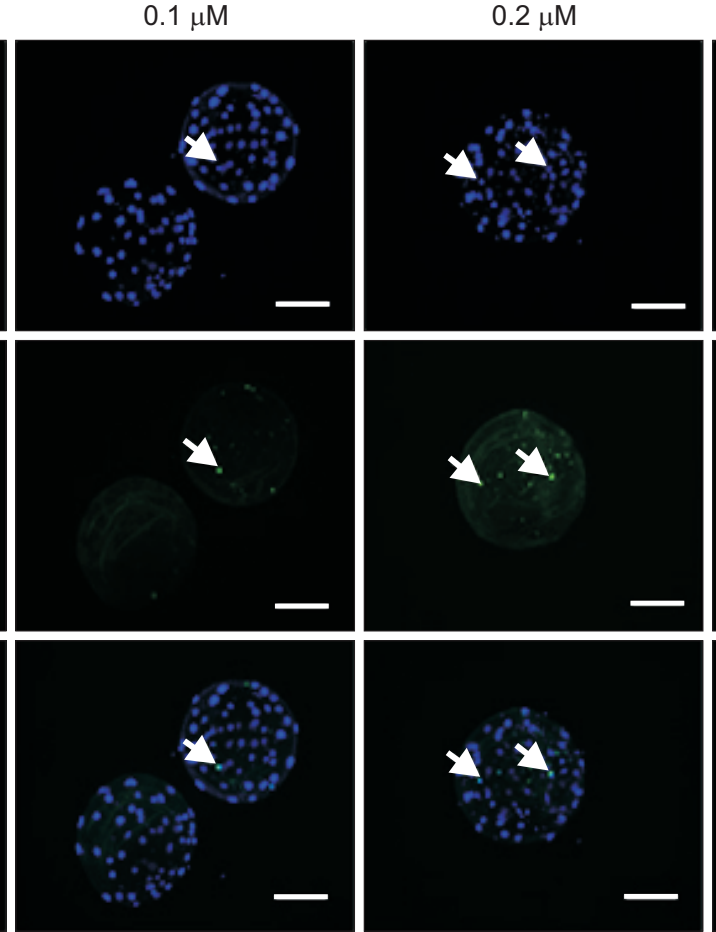

\begin{tabular}{cc} 
GT1b \\
\hline $0.1 \mu \mathrm{M}$ & $0.2 \mu \mathrm{M}$
\end{tabular}
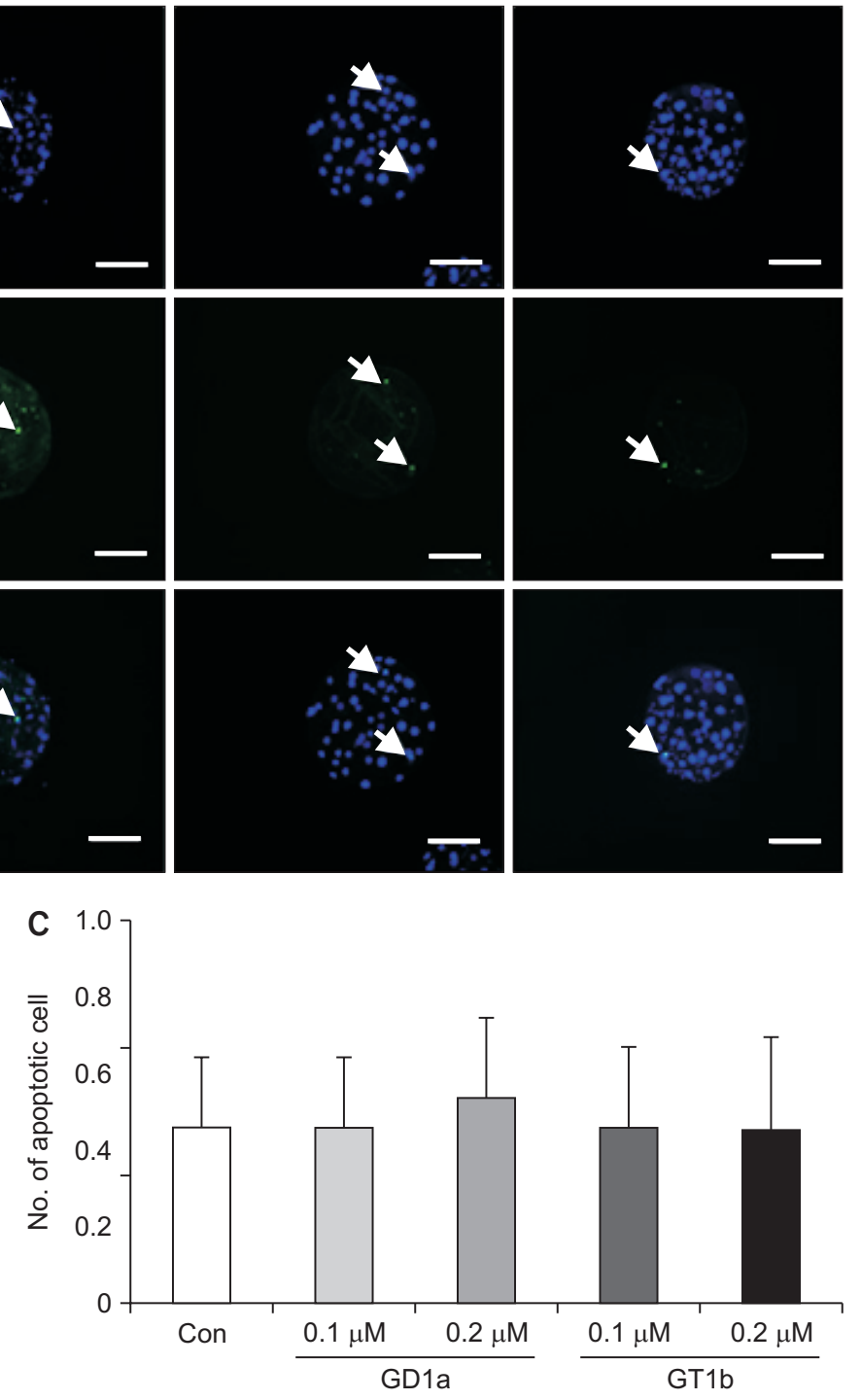

Fig. 4. Apoptotic patterns in blastocysts derived from GD1a and GT1b treated embryos. Total chromatin content were determined by staining with DAPI (blue) and fragmented DNA was labeled via terminal deoxynucleotidyl transferase-mediated dUTP nick-end labeling (TUNEL; green, white arrows). Merged images show TUNEL and DAPI signals. Scale bar $=100 \mu \mathrm{m}$. Data in the bar graph represent the means \pm SEM of three independent experiments. Differences were considered significant at ${ }^{*} p<0.05$ and ${ }^{* *}<0.01$.

Table 4. Effect of ganglioside GD1a and GT1b on total cell number and apoptosis in blastocysts

\begin{tabular}{lcccc}
\hline \multirow{2}{*}{ Group } & No. of embryos examined & \multicolumn{2}{c}{ No. of cells } & TUNEL-positive cells (\%) \\
\cline { 3 - 4 } & & Total & TUNEL-positive & \\
\hline Con & 14 & $33.4 \pm 11.3$ & $0.1 \pm 0.3$ & $0.8 \pm 0.3$ \\
GD1a 0.1 $\mu \mathrm{M}$ & 16 & $47.6 \pm 16.2$ & $0.2 \pm 0.4$ & $0.3 \pm 0.6$ \\
GD1a 0.2 $\mu \mathrm{M}$ & 16 & $34.6 \pm 12.0$ & $0.1 \pm 0.3$ & $0.3 \pm 0.9$ \\
GT1b 0.1 $\mu \mathrm{M}$ & 10 & $44.6 \pm 13.0$ & $0.2 \pm 0.4$ & $0.4 \pm 0.9$ \\
GT1b 0.2 $4 \mathrm{M}$ & $37.9 \pm 14.7$ & $0.2 \pm 0.4$ & $0.4 \pm 0.8$ \\
\hline
\end{tabular}

This experiment was replicated three times. Data are the mean \pm SD. 
A

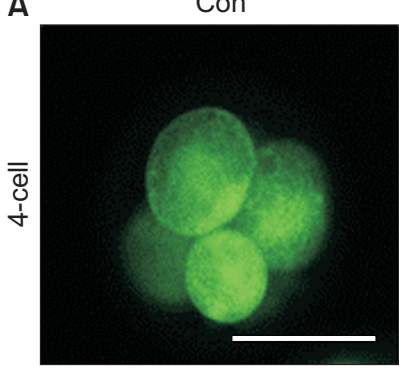

B

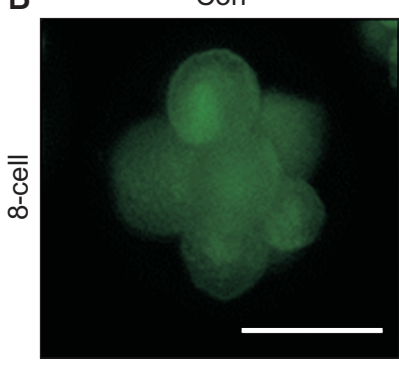

C

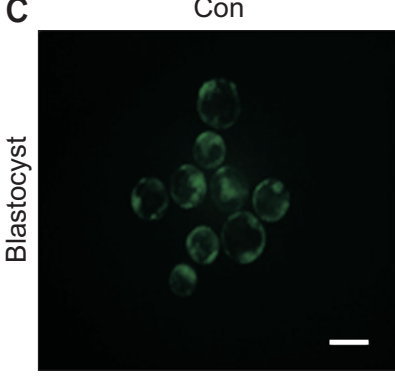

GD1a $0.1 \mu \mathrm{M}$

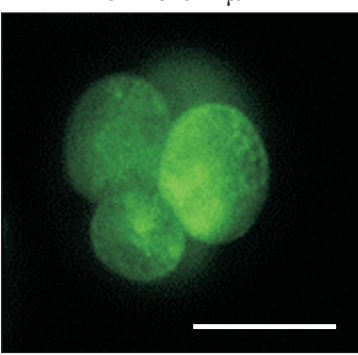

GD1a $0.1 \mu \mathrm{M}$

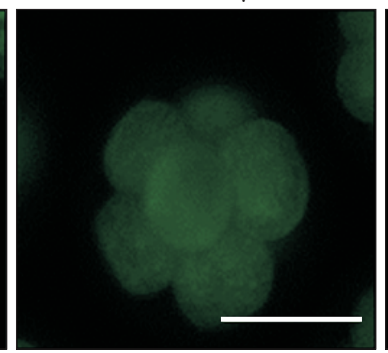

GD1a $0.1 \mu \mathrm{M}$

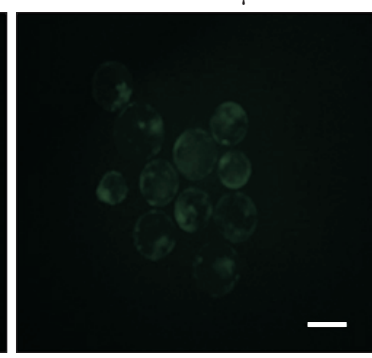

GT1b $0.1 \mu \mathrm{M}$

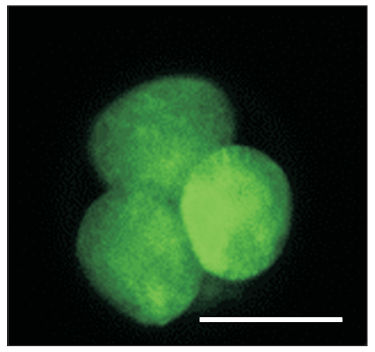

GT1b $0.1 \mu \mathrm{M}$

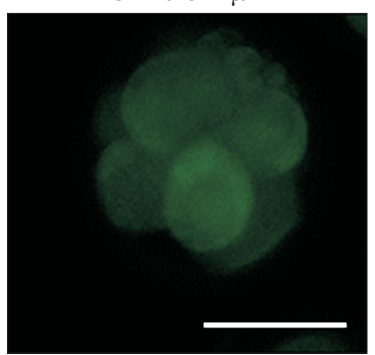

GT1b $0.1 \mu \mathrm{M}$

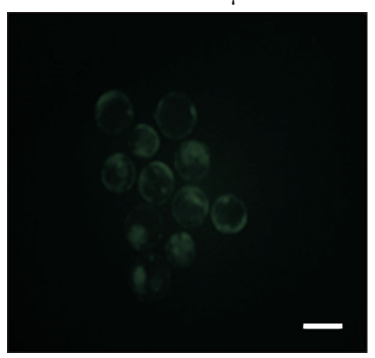

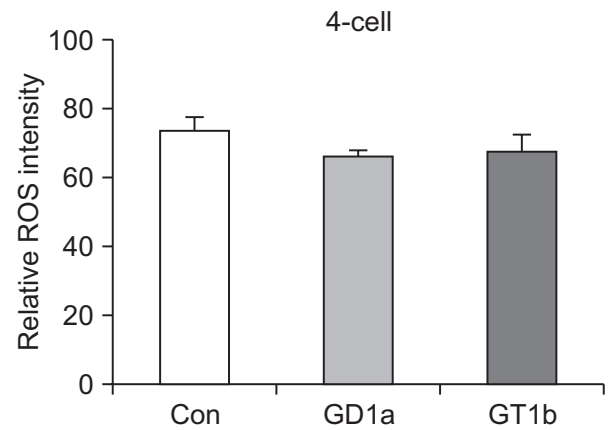

8-cell

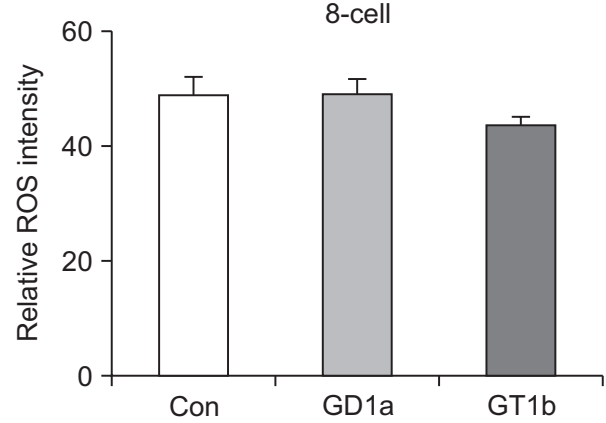

Blastocyst

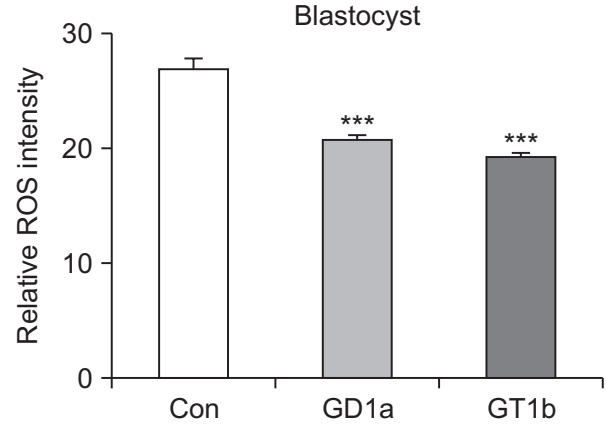

Fig. 5. Detection of ROS levels in blastocysts derived from ganglioside GD1a and GT1b treated embryos by H2DCFDA staining. (A) Fluorescence imaging of ROS production in blastocysts by H2DCFDA. (B) Quantification of ROS levels in blastocysts. Scale bar $=100 \mu \mathrm{m}$. Data in the bar graph represent the means \pm SEM of three independent experiments. Differences were considered significant at ${ }^{* * *} p<0.001$.

GD1a and GT1b treated blastocyst stage. Therefore, we first identified possible roles of ganglioside GD1a and GT1b in an antioxidative effect during in vitro culture (IVC) of porcine embryos. Interestingly, the results above showed that ganglioside GD1a and GT1b play major role as ROS scavenger in early embryo development of pigs.

Ganglioside GD1a and GT1b are known to be synthesized by the ST3GAL2 enzyme (Sturgill et al., 2012). In previous studies, in ST3GAL2 knockout(K/O) mice, the synthesis of GD1a and GT1b was decreased and the expression of GM1a and GD1b was increased (Sturgill et al., 2012). Fig. 1 shows expression patterns of ST3GAL2. To infer the expression of GD1a and GT1b in embryos development stages, we measured the expression of ST-
3GAL2 using IF staining. The red fluorescence expression of ST3GAL2 increased at the 4-cells and 8 cells stage of porcine embryos after IVF. Therefore, we speculated that there is a close correlation between GD1a and early embryonic development. Based on these results, we performed subsequent experiments using GD1a and GT1b treated culture medium during IVC.

Ganglioside GD1a and GT1b regulate cellular function, such as cell differentiation and growth control in neuron (Magistretti et al., 2019) and cancer cells (Groux-Degroote et al., 2018). Our previous study shows that GD1a can improve porcine oocyte quality, maturation capacity, and subsequent embryonic development through its various biological activities such as EGFR activation or ERK 
signaling (Kim et al., 2016; Park et al., 2017). In previous study, we confirmed that GT1b was expressed during the embryonic development in mice (Kim et al., 2008). However, effects of GD1a and GT1b treatment on embryos development in mammalian embryos has not investigated. Therefore, we examined the effects of GD1a and GT1b on embryos development in porcine embryos during IVC. We confirmed that the development rate of porcine embryos was significantly increased in the $0.1 \mu \mathrm{M}$ GD1a and GT1b treated culture medium (Fig. 2 and 3). We first proved that GD1a and GT1b enhance porcine embryos development.

Apoptosis has been used as an important indicator for embryonic development and embryonic loss following the stress response of cells in IVP embryos. Previous studies have reported that the rate of apoptosis was increased by harmful substances such as $\mathrm{H}_{2} \mathrm{O}_{2}$ during embryos development (Lee et al., 2015). On the other hand, the rate of apoptosis was decreased in the melatonin and antioxidants treated groups (Do et al., 2015; Lee et al., 2015). Therefore, apoptosis in blastocysts is an important indicator for evaluation the quality of blastocysts and in vitro environments. In addition, we investigated that the ganglioside GM3 is involved the apoptotic stress in porcine oocyte and embryo during in vitro production (IVP) progression (Chae et al., 2015; Kim et al., 2018). However, no reports have identified this crosstalk between GD1a or GT1b and blastocyst development capacity in porcine embryos. In our results, there was no significant difference in the number of total cells and ratio of apoptosis rate. However, we confirmed that the total cell number was increased in $0.1 \mu \mathrm{M}$ GD1a and GT1b treated groups (Table 4). This result support that the GD1a and GT1b treatment improves blastocyst quality by protecting DNA damage mediated cellular apoptosis.

ROS are produced during normal cell activation and are involved in a variety of biological processes, including cell differentiation and gene expression. Therefore, maintaining homeostasis of ROS is very important for cell growth, survival (Covarrubias et al., 2008), embryo development, and cloned embryos production (Fang et al., 2018). Oxidative stress is a phenomenon in which the ROS in the cell increases due to an imbalance between the production of ROS and the antioxidant reactions that remove it, causing it to react with and damage DNA, proteins, and lipids (Tarín, 1996). This damage is known to cause many cellular problems on embryos development during IVP of cow (Favetta et al., 2007) and mice (Goto et al., 1993). Our previous results have reported increase in the efficiency of IVF embryos production and development potential using natural substances or antioxidants to suppress ROS or oxidative stress in pigs (Lee et al., 2015; Yang et al., 2018). According to previous studies, Ganglioside GT1b protected spermatozoa against $\mathrm{H}_{2} \mathrm{O}_{2}$ damage in human (Gavella et al., 2012; Gavella et al., 2013), and reduced ROS during in vitro maturation of porcine oocytes (Hwang et al., 2015). Also, Ganglioside GD1a reduced ROS induced by LPS and increased survival rate in PC12 cells (Nikolaeva et al., 2015). In present study, there was no significant difference in intracellular ROS levels between GD1a and GT1b treated groups and control group at the cleavage stage (4-8 cells). However, in the blastocyst stage, intracellular ROS levels were significantly decreased in the GD1a and GT1b treated groups compared with control group (Fig. 5). These results suggest that ganglioside GD1a and GT1b decrease intracellular ROS levels of porcine blastocysts in vitro.

\section{CONCLUSION}

In conclusion, the results of the present study suggest that treatment of ganglioside GD1a and GT1b in porcine preimplantation embryos enhanced rate of blastocyst development. Although there was no significant difference in the rate of apoptosis, number of total cell was increased. Furthermore, we confirmed the intracellular ROS levels decreased in the blastocyst stage. Therefore, we suggest that treatment of ganglioside GD1a and GT1b during IVC may assist in the preimplantation development of porcine embryos.

\section{CONFLICTS OF INTEREST}

No potential conflict of interest relevant to this article was reported.

\section{ACKNOWLEDGEMENTS}

This research was supported by the Basic Science Research Program through the National Research Foundation of Korea (NRF-2018R1C1B002922 and NRF2019R1A2C1085199) funded by the Ministry of Education and the Ministry of Science and ICT, Republic of Korea. 


\section{AUTHOR CONTRIBUTIONS}

Conceptualization: Jin-Woo Kim, Hyo-Jin Park and DeogBon Koo

Data curation: Jin-Woo Kim and Seul-Gi Yang

Formal analysis: Jin-Woo Kim, Seul-Gi Yang, Hyo-Jin Park

Supervision and funding acquisition: Deog-Bon Koo

Investigation: Jin-Woo Kim and Seul-Gi Yang

Methodology: Jin-Woo Kim, Seul-Gi Yang and Hyo-Jin Park

Resources, Project administration: Jin-Woo Kim, Seul-

Gi Yang and Deog-Bon Koo

Validation: Jin-Woo Kim and Seul-Gi Yang

Writing - original draft: Jin-Woo Kim and Hyo-Jin Park

Writing - review \& editing: Seul-Gi Yang, Hyo-Jin Park and Deog-Bon Koo

\section{AUTHOR'S POSITION AND ORCID NO.}

\author{
JW Kim, PhD, \\ https://orcid.org/0000-0002-0089-680X \\ HJ Park, PhD, \\ https://orcid.org/0000-0003-3287-2914 \\ SG Yang, PhD Candidate, \\ https://orcid.org/0000-0003-3247-2443 \\ DB Koo, Professor, \\ https://orcid.org/0000-0001-7825-9598
}

\section{REFERENCES}

Chae SK, Park HJ, Kim JW, Ahn JH, Park SY, Park JY, Yang SG, Koo DB. 2015. Changes of ganglioside GM3 expression in porcine oocyte maturation and early embryonic development in vitro. J. Emb. Trans. 30:319-325.

Chen PR, Redel BK, Spate LD, Ji T, Salazar SR, Prather RS. 2018. Glutamine supplementation enhances development of in vitro-produced porcine embryos and increases leucine consumption from the medium. Biol. Reprod. 99:938-948.

Choi J, Park SM, Lee E, Kim JH, Jeong YI, Lee JY, Park SW, Kim HS, Hossein MS, Jeong YW, Kim S, Hyun SH, Hwang WS. 2008. Anti-apoptotic effect of melatonin on preimplantation development of porcine parthenogenetic embryos. Mol. Reprod. Dev. 75:1127-1135.

Covarrubias L, Hernández-García D, Schnabel D, Salas-Vidal E, Castro-Obregón S. 2008. Function of reactive oxygen species during animal development: passive or active? Dev. Biol. 320:1-11.

Crow JP. 1997. Dichlorodihydrofluorescein and dihydrorhodamine 123 are sensitive indicators of peroxynitrite in vitro: implications for intracellular measurement of reactive nitro- gen and oxygen species. Nitric Oxide 1:145-157.

Do LT, Shibata Y, Taniguchi M, Nii M, Nguyen TV, Tanihara F, Takagi M, Otoi T. 2015. Melatonin supplementation during in vitro maturation and development supports the development of porcine embryos. Reprod. Domest. Anim. 50:1054-1058.

Fang X, Qamar AY, Yoon KY, Cho J. 2018. Improved preimplantation development of porcine cloned embryos by flavone supplement as antioxidant. J. Emb. Trans. 33:255-264.

Favetta LA, St John EJ, King WA, Betts DH. 2007. High levels of p66shc and intracellular ROS in permanently arrested early embryos. Free Radic. Biol. Med. 42:1201-1210.

Gavella M and Lipovac V. 2013. Protective effects of exogenous gangliosides on ROS-induced changes in human spermatozoa. Asian J. Androl. 15:375-381.

Gavella M, Lipovac V, Garaj-Vrhovac V, Gajski G. 2012. Protective effect of gangliosides on DNA in human spermatozoa exposed to cryopreservation. J. Androl. 33:1016-1024.

Goto Y, Noda Y, Mori T, Nakano M. 1993. Increased generation of reactive oxygen species in embryos cultured in vitro. Free Radic. Biol. Med. 15:69-75.

Groux-Degroote S, Rodríguez-Walker M, Dewald JH, Daniotti JL, Delannoy P. 2018. Gangliosides in cancer cell signaling. Prog. Mol. Biol. Transl. Sci. 156:197-227.

Hwang SU, Jeon Y, Yoon JD, Cai L, Kim E, Yoo H, Kim KJ, Park KM, Jin M, Kim H, Hyun SH. 2015. Effect of ganglioside GT1b on the in vitro maturation of porcine oocytes and embryonic development. J. Reprod. Dev. 61:549-557.

Kim BH, Jung JU, Ko K, Kim WS, Kim SM, Ryu JS, Jin JW, Yang HJ, Kim JS, Kwon HC, Nam SY, Kwak DH, Park YI, Koo DB, Choo YK. 2008. Expression of ganglioside GT1b in mouse embryos at different developmental stages after cryopreservation. Arch. Pharm. Res. 31:88-95.

Kim JW, Park HJ, Chae SK, Ahn JH, Do GY, Choo YK, Park JJ, Jung BD, Kim SU, Chang KT, Koo DB. 2016. Ganglioside GDla promotes oocyte maturation, furthers preimplantation development, and increases blastocyst quality in pigs. J. Reprod. Dev. 62:249-255.

Kim JW, Park HJ, Jung JM, Yang SG, Kim MJ, Kim IS, Jegal HG, Koo DB. 2018. Reversible effects of exogenous GM3 on meiotic maturation and cumulus cells expansion of porcine cumulus-oocyte complexes. J. Emb. Trans. 33:287-296.

Kim SM, Jung JU, Ryu JS, Jin JW, Yang HJ, Ko K, You HK, Jung KY, Choo YK. 2008. Effects of gangliosides on the differentiation of human mesenchymal stem cells into osteoblasts by modulating epidermal growth factor receptors. Biochem. Biophys. Res. Commun. 371:866-871.

Kwak DH, Seo BB, Chang KT, Choo YK. 2011. Roles of gangliosides in mouse embryogenesis and embryonic stem cell differentiation. Exp. Mol. Med. 43:379-388.

Lee E, Min SH, Song BS, Yeon JY, Kim JW, Bae JH, Park SY, Lee YH, Kim SU, Lee DS, Chang KT, Koo DB. 2015. Exogenous $\gamma$-tocotrienol promotes preimplantation development and improves the quality of porcine embryos. Reprod. Fertil. Dev. 27:481-490.

Macháty Z, Day BN, Prather RS. 1998. Development of early 
porcine embryos in vitro and in vivo. Biol. Reprod. 59:451455.

Magistretti PJ, Geisler FH, Schneider JS, Li PA, Fiumelli H, Sipione S. 2019. Gangliosides: treatment avenues in neurodegenerative disease. Front. Neurol. 10:859.

Nikolaeva S, Bayunova L, Sokolova T, Vlasova Y, Bachteeva V, Avrova N, Parnova R. 2015. GM1 and GDla gangliosides modulate toxic and inflammatory effects of E. coli lipopolysaccharide by preventing TLR4 translocation into lipid rafts. Biochim. Biophys. Acta. 1851:239-247.

Park HJ, Kim JW, Park JY, Yang SG, Jung JM, Kim MJ, Koo DB. 2017. Ganglioside GDla activates the phosphorylation of EGFR in porcine oocytes maturation in vitro. J. Emb. Trans. 32:17-24.

Prather RS, Hawley RJ, Carter DB, Lai L, Greenstein JL. 2003. Transgenic swine for biomedicine and agriculture. Theriogenology 59:115-123.

Rath D, Niemann H, Torres CR. 1995. In vitro development to blastocysts of early porcine embryos produced in vivo or in vitro. Theriogenology 43:913-926.

Sturgill ER, Aoki K, Lopez PH, Colacurcio D, Vajn K, Lorenzini I, Majić S, Yang WH, Heffer M, Tiemeyer M, Marth JD, Schnaar RL. 2012. Biosynthesis of the major brain gangliosides GDla and GT1b. Glycobiology 22:1289-1301.

Tarín JJ. 1996. Potential effects of age-associated oxidative stress on mammalian oocytes/embryos. Mol. Hum. Reprod. 2:717-724.

Yang HW, Hwang KJ, Kwon HC, Kim HS, Choi KW, Oh KS. 1998. Detection of reactive oxygen species (ROS) and apoptosis in human fragmented embryos. Hum. Reprod. 13:998-1002.

Yang SG, Park HJ, Kim JW, Jung JM, Kim MJ, Jegal HG, Kim IS, Kang MJ, Wee G, Yang HY, Lee YH, Seo JH, Kim SU, Koo DB. 2018. Mito-TEMPO improves development competence by reducing superoxide in preimplantation porcine embryos. Sci. Rep. 8:10130. 